Research Article

\title{
Computation of the Double Metric Dimension in Convex Polytopes
}

\author{
Liying Pan $\left(\mathbb{D},{ }^{1}\right.$ Muhammad Ahmad $\mathbb{D}^{2},{ }^{2}$ Zohaib Zahid $\mathbb{D}^{,},{ }^{2}$ and Sohail Zafar ${ }^{2}$ \\ ${ }^{1}$ Department of Mathematics, Baoji Education Institute of Shaanxi, Baoji 721004, China \\ ${ }^{2}$ University of Management and Technology (UMT), Lahore, Pakistan \\ Correspondence should be addressed to Zohaib Zahid; zohaib_zahid@hotmail.com
}

Received 18 March 2021; Accepted 24 September 2021; Published 26 October 2021

Academic Editor: Kenan Yildirim

Copyright (c) 2021 Liying Pan et al. This is an open access article distributed under the Creative Commons Attribution License, which permits unrestricted use, distribution, and reproduction in any medium, provided the original work is properly cited.

A source detection problem in complex networks has been studied widely. Source localization has much importance in order to model many real-world phenomena, for instance, spreading of a virus in a computer network, epidemics in human beings, and rumor spreading on the internet. A source localization problem is to identify a node in the network that gives the best description of the observed diffusion. For this purpose, we select a subset of nodes with least size such that the source can be uniquely located. This is equivalent to find the minimal doubly resolving set of a network. In this article, we have computed the double metric dimension of convex polytopes $R_{n}$ and $Q_{n}$ by describing their minimal doubly resolving sets.

\section{Introduction and Preliminaries}

Let $G$ be a finite and connected graph with vertex set $V_{G}$ and edge set $E_{G}$. The cardinality of $V_{G}$ and $E_{G}$ is called the order and the size of the graph $G$, respectively. For any two vertices $b$ and $c$ of graph $G$, the number of edges in a shortest path between the vertices $b$ and $c$ is called the distance between them and is represented as $d(b, c)$. A vertex $b \in V_{G}$ is said to resolve two vertices $c$ and $e$ if $d(b, c)$ is not equal to $d(b, e)$. Consider $P=\left\{p_{i} \mid 1 \leq i \leq k\right\} \subseteq V_{G}$ be an ordered set and $v$ be a vertex of $G$; then, the representation $r(v, P)$ of $v$ with respect to $P$ is the $k$-vector $\left(d\left(v, p_{i}\right)\right)_{i=1}^{k}$. The set $P$ is called a resolving set for graph $G$ if any vertex of $G$ has unique representation with respect to $P$. A resolving set having least number of vertices is called a metric basis of $G$. $\operatorname{dim}(G)$ represents cardinality of metric basis and is known as the metric dimension of $G$. For the notions that are used and not defined in this paper, we refer $[1,2]$.

In graph theory, the notion of minimal resolving sets were first defined in $[3,4]$ by Slater, Harary, and Melter independently. Slater used the metric dimension as location number of the graph. He used this concept by placing sonar/ loran detecting devices with the least cardinality in such a way that the location of each vertex is unique with respect to its distances from the devices in the network. The idea of trilateration in real two-dimensional plane can be generalized in terms of the metric dimension of graphs. For instance, in Global Positioning System (GPS), three satellites in orbit uniquely determine the location of an object on earth by using distances. Also, for the theoretical study of the metric dimension, there are many inspirational applications regarding robot navigation [5] and chemical structure [6]. Different coin weighing problems discussed in $[7,8]$ and complete analysis of Mastermind game found in [9] have strong connections with the metric dimension of hamming graphs. Many applications of this concept have been explored in different fields such as geographical routing protocols [10], combinatorial optimization [11], network discovery and verification [12], pharmaceutical chemistry [6], etc. [13].

It is a computationally difficult problem to determine the exact value of the metric dimension of arbitrary graphs. Therefore, some useful bounds have been found for different classes of graphs.

Chartrand et al. [6], in 2000, characterized all graphs with metric dimension $n-1, n-2$, and 1 . Buczkowski et al. [14] and Tomescu and Javaid [15] determined the metric dimension of wheel graphs and Jahangir graphs, respectively. Ali et al. [16] studied the metric dimension of Mobius 
ladders. They proved that Mobius ladders constitute a family of cubic graphs with constant metric dimension. Baca et al. [17] computed the metric dimension of regular bipartite graphs. Imran and Baig $[18,19]$ determined the metric dimension for some classes of convex polytopes. In 2015, Tomescu and Imran used $R$-sets for the computation of the metric dimension of necklace graphs [20]. Ahmad et al. computed the metric dimension for the families of kayak paddle graphs and chorded cycles (for details, see [21-23]).

It is an interesting challenge in complex networks to localize the epidemic source. For example, in order to find a mysterious source of virus spreading throughout the network, the only information needed to identify the mysterious source is the infection times of the subset of vertices known as sensors. These sensors may record their least time of being infected. Now, the main problem consists of determining how many sensors are needed to assure that the virus source is exactly located. The characteristic known as the double metric dimension is the answer of this problem (see [24]).

The recognition of the virus source might be straightforward if one can notice the whole procedure of the spreading of virus. Unfortunately, the whole procedure might be very expensive because of the cost of collecting information. If the starting time of epidemic spread is not known, in that case accurate recognition of infection source is possible if and only if the network sensor set is a doubly resolving set.

Cáceres et al. presented the doubly resolving sets by demonstrating its relation with the metric dimension of the Cartesian product of the graph $G$ and also proved that the least cardinality of a doubly resolving set is an upper bound of the metric dimension of the graph under consideration (see [25]). For $a_{1}, a_{2}, u_{1}, u_{2} \in V_{G}, a_{1}$ and $a_{2}$ are said to doubly resolve $u_{1}$ and $u_{2}$ if $d\left(u_{1}, a_{1}\right)-d\left(u_{1}, a_{2}\right) \neq d\left(u_{2}\right.$, $\left.a_{1}\right)-d\left(u_{2}, a_{2}\right)$. If any two vertices of $G$ are doubly resolved by some two vertices in $D \subseteq V_{G}$, then $D$ is termed doubly resolving set of $G$. In other words, there do not exist $u_{1}, u_{2} \in V_{G}$ such that $r\left(u_{1} \mid D\right)-r\left(u_{2} \mid D\right) \neq m \cdot u$ for some $m \in \mathbb{Z}$, and $u$ is a vector of length $|D|$ with all entries equal to 1. Such $D$ of least cardinality is known as minimal doubly resolving set and its cardinality is called the double metric dimension of $G$ represented by $\psi(G)$. It should be noted that, for $a_{1}, a_{2} \in D$ and $u_{1}, u_{2} \in V_{G}, d\left(u_{1}, a_{1}\right)-d\left(u_{2}, a_{1}\right) \neq 0$ or $d\left(u_{1}, a_{2}\right)-d\left(u_{2}, a_{2}\right) \neq 0$ implies $D$ to be also a resolving set. Consequently, for any graph $G$, we have $\operatorname{dim}(G) \leq \psi(G)$. The NP-hardness of $\operatorname{dim}(G)$ and $\psi(G)$ were proven by Khuller et al. in [5] and Kratica et al. in [26], respectively.

In [24], it was discussed that to identify the virus source in a star-like network is more difficult compared to that in a path-like network. A star graph with $n$ vertices must have a doubly resolving set consisting of all the external vertices, which show the double metric dimension is $n-1$, and for the path graph, it is 2 . This also implies that the double metric dimension always depends upon the topology of network.

In [27], the metric and double metric dimensions were found to be equal in case for some families of the circulant graph. Prism graphs, hamming graphs, and some convex polytopes have been discussed in the context of the double metric dimension in [28-30]. Ahmad et al. determined the minimal doubly resolving sets for some families of Harary graphs (see [31]). Chen et al. [32] provided the first explicit approximation lower and upper bounds for the minimum doubly resolving set problem. In $[33,34]$, Ahmad et al. have determined the metric and double metric dimensions for the line graphs of prism graphs, $n$-sunlet graphs, and kayak paddle graphs.

The convex polytopes in the context of metric dimension had been studied widely by many authors in last few years. The computation of the double metric dimension in convex polytopes is a challenging problem nowadays. In this paper, we compute the double metric dimensions of convex polytopes $R_{n}$ and $Q_{n}$ defined by Baca in [35]. Imran et al. [36] determined the metric dimensions of these convex polytopes given in the following theorems:

Theorem 1. Let $R_{n}$ be the graph of convex polytopes; then, for every $n \geq 5, \operatorname{dim}\left(R_{n}\right)=3$.

Theorem 2. Let $Q_{n}$ be the graph of convex polytopes; then, for every $n \geq 6, \operatorname{dim}\left(Q_{n}\right)=3$.

The above results are also useful in finding the lower bound of the double metric dimensions of convex polytopes $R_{n}$ and $Q_{n}$. follows.

The remaining portion of the article is structured as

The double metric dimension for the convex polytopes $R_{n}$ and $Q_{n}$ is described in Sections 2 and 3, respectively. Finally, we conclude in Section 4 that the double metric dimension for the convex polytopes $R_{n}$ and $Q_{n}$ is constant.

\section{The Double Metric Dimension for the Convex Polytope $R_{n}$}

This section will particularly focus to find out the double metric dimension for convex polytope $R_{n}$. The graph of convex polytope $R_{n}$ consists of three-sided, four-sided, and $n$-sided faces, as illustrated in Figure 1.

We label the vertices of the inner cycle by $\left\{e_{j}: \forall 0 \leq\right.$ $j \leq n-1\}$, the vertices of the center cycle by $\left\{f_{j}: \forall 0 \leq j \leq\right.$ $n-1\}$, and the vertices of the outer cycle by $\left\{g_{j}: \forall 0 \leq\right.$ $j \leq n-1\}$, as demonstrated in Figure 1 .

Theorem 1 implies that $\psi\left(R_{n}\right) \geq 3$, for $n \geq 5$. Furthermore, we will show that $\psi\left(R_{n}\right)=3$, for $n \geq 5$. Now, to calculate distances for the convex polytope $R_{n}$, define $S_{j}\left(f_{0}\right)=$ $\left\{f \in V_{R_{n}}: d\left(f_{0}, f\right)=j\right\}$ is the set of vertices in $V_{R_{n}}$ at distance $j$ from $f_{0}$. For $\psi\left(R_{n}\right)$ with $n \geq 5$, we can find the sets $S_{j}\left(f_{0}\right)$ that are elaborated in Table 1. Obviously, it can be seen from Figure 1 that $S_{j}\left(f_{0}\right)=\varnothing$, when $j>((n+2) / 2)$, for even $n$ and $S_{j}\left(f_{0}\right)=\varnothing$, when $j>((n+1) / 2)$, for odd $n$. Note that the sets $S_{j}\left(f_{0}\right)$ can clearly be used to find the distance between the two arbitrary vertices of $V_{R_{n}}$ as follows.

The symmetry in Figure 1 shows that 


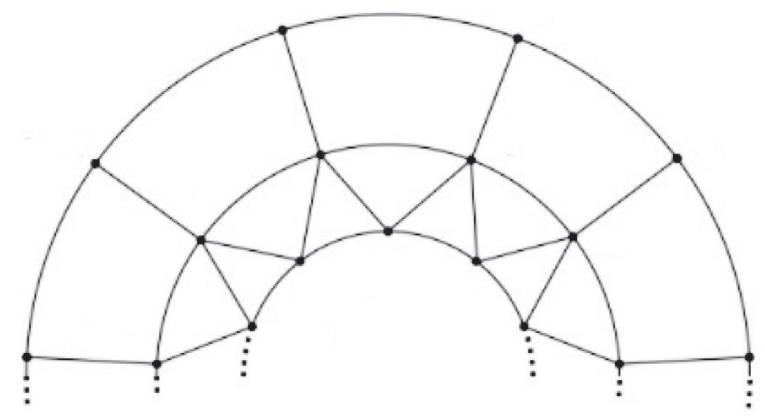

Figure 1: Convex polytope $R_{n}$.

TABle 1: $S_{j}\left(f_{0}\right)$ for $R_{n}$.

\begin{tabular}{|c|c|c|}
\hline$n$ & $j$ & $S_{j}\left(f_{0}\right)$ \\
\hline Even & $\begin{array}{c}1 \\
2 \leq j \leq\lfloor n / 2\rfloor-1 \\
n / 2 \\
(n+2) / 2\end{array}$ & $\begin{array}{c}\left\{e_{0}, e_{n-1}, f_{1}, f_{n-1}, g_{0}\right\} \\
\left\{e_{j-1}, e_{n-j}, f_{j}, f_{n-j}, g_{j-1}, g_{n-j+1}\right\} \\
\left\{e_{(n-2) / 2}, e_{n / 2}, f_{n / 2}, g_{(n-2) / 2}, g_{(n+2) / 2}\right\} \\
\left\{g_{n / 2}\right\}\end{array}$ \\
\hline Odd & $\begin{array}{l}(n-1) / 2 \\
(n+2) / 2\end{array}$ & $\begin{array}{c}\left\{e_{(n-3) / 2}, e_{(n+1) / 2}, f_{(n-1) / 2}, f_{(n+1) / 2}, g_{(n-3) / 2}, g_{(n+3) / 2}\right\} \\
\left\{e_{(n-1) / 2}, g_{(n-1) / 2}, g_{(n+1) / 2}\right\}\end{array}$ \\
\hline
\end{tabular}

For odd values of $n$, we have

$$
\begin{aligned}
& d\left(f_{j}, e_{k}\right)= \begin{cases}d\left(f_{0}, e_{|j-k|}\right)-1, & \text { if } 1 \leq|j-k| \leq \frac{n-1}{2} \text { for } j>k, \\
d\left(f_{0}, e_{|j-k|}\right)+1, & \text { if } \frac{n+1}{2} \leq|j-k| \leq n-1 \text { for } j>k, \\
d\left(f_{0}, e_{|j-k|}\right), & \text { if } 0 \leq|j-k| \leq n-1 \text { for } k \geq j,\end{cases} \\
& d\left(e_{j}, g_{k}\right)= \begin{cases}d\left(f_{0}, g_{|j-k|}\right)+1, & \text { if } 0 \leq|j-k| \leq \frac{n-1}{2} \text { for } j \geq k, \\
d\left(f_{0}, g_{|j-k|}\right), & \text { if } \frac{n+1}{2} \leq|j-k| \leq n-1 \text { for } j>k, \\
d\left(f_{0}, g_{|j-k|}\right), & \text { if } 1 \leq|j-k| \leq \frac{n-1}{2} \text { for } k>j, \\
d\left(f_{0}, g_{|j-k|}\right)+1, & \text { if } \frac{n+1}{2} \leq|j-k| \leq n-1 \text { for } k>j,\end{cases} \\
& d\left(e_{j}, e_{k}\right)= \begin{cases}d\left(f_{0}, e_{|j-k|}\right)-1, & \text { if } 0 \leq|j-k| \leq \frac{n-1}{2}, \\
d\left(f_{0}, e_{|j-k|}\right), & \text { if } \frac{n+1}{2} \leq|j-k| \leq n-1 .\end{cases}
\end{aligned}
$$


For even values of $n$, we have

$$
d\left(f_{j}, e_{k}\right)= \begin{cases}d\left(f_{0}, e_{|j-k|}\right)-1, & \text { if } 1 \leq|j-k| \leq \frac{n-2}{2} \text { for } j>k, \\ d\left(f_{0}, e_{|j-k|}\right), & \text { if }|j-k|=\frac{n}{2} \text { for } j>k, \\ d\left(f_{0}, e_{|j-k|}\right)+1, & \text { if } \frac{n+2}{2} \leq|j-k| \leq n-1 \text { for } j>k, \\ d\left(f_{0}, e_{|j-k|}\right), & \text { if } 0 \leq|j-k| \leq n-1 \text { for } k \geq j, \\ d\left(f_{0}, e_{|j-k|}\right)-1, & \text { if } 0 \leq|j-k| \leq \frac{n-2}{2}, \\ d\left(f_{0}, e_{|j-k|}\right), & \text { if } \frac{n}{2} \leq|j-k| \leq n-1, \\ d\left(f_{0}, g_{|j-k|}\right), & \text { if } \frac{n}{2} \leq|j-k| \leq n-1 \text { for } j>k .\end{cases}
$$

As an outcome, if the distance $d\left(f_{0}, f\right)$ is known for each $f \in V_{R_{n}}$, then one can recreate the distances between any two vertices of $V_{R_{n}}$.

Lemma 1. $\psi\left(R_{n}\right)=3$ for $n \geq 5$, whenever $n$ is even.

Proof. According to the set $D=\left\{e_{0}, e_{(n-2) / 2}, g_{1}\right\}$, the representations of $R_{n}$ is explained in Table 2 .

Now, from Table 2, the first metric coordinate of the vector of $f_{0} \in S_{j}\left(f_{0}\right)$ is equal to 1 . Similarly, it can be observed that there are no two vertices $h_{1}, h_{2} \in S_{j}\left(f_{0}\right)$, for every $j \in\{1,2, \ldots,((n+2) / 2)\}$ such as $r\left(h_{1}, D\right)-r\left(h_{2}\right.$, $D)=0$. Thus, there are no two vertices $h_{1} \in S_{j}\left(f_{0}\right)$ and $h_{2} \in S_{k}\left(f_{0}\right)$, for any $j, k \in\{1,2, \ldots,((n+2) / 2)\}, j \neq k$, such as $r\left(h_{1}, D\right)-r\left(h_{2}, D\right)=j-k$. Therefore, the set $D=\left\{e_{0}\right.$, $\left.e_{(n-2) / 2}, g_{1}\right\}$ is the minimal doubly resolving set. Hence, Lemma 1 holds.

Lemma 2. $\psi\left(R_{n}\right)=3$, for $n \geq 5$, whenever $n$ is odd.

Proof. The minimal doubly resolving sets for $R_{5}$ and $R_{7}$ are

$$
D= \begin{cases}\left\{e_{0}, e_{2}, g_{4}\right\}, & \text { if } n=5 \\ \left\{e_{0}, e_{3}, g_{2}\right\}, & \text { if } n=7\end{cases}
$$

Now, Table 3 demonstrates that the representations of $R_{n}($ where $n \geq 9)$ in relation to the set $D=\left\{e_{0}, e_{(n-1) / 2}\right.$, $\left.g_{(n+5) / 2}\right\}$.

Now, from Table 3, the first metric coordinate of the vector of $f_{0} \in S_{j}\left(f_{0}\right)$ is equal to 1 . Similarly, it can be observed that there are no two vertices $h_{1}, h_{2} \in S_{j}\left(f_{0}\right)$, for every $j \in\{1,2, \ldots,((n+1) / 2)\}$ such as $r\left(h_{1}, D\right)-r\left(h_{2}\right.$, $D)=0$. Thus, there are no two vertices $h_{1} \in S_{j}\left(f_{0}\right)$ and $h_{2} \in S_{k}\left(f_{0}\right)$, for any $j, k \in\{1,2, \ldots,((n+1) / 2)\}, j \neq k$, such as $r\left(h_{1}, D\right)-r\left(h_{2}, D\right)=j-k$. Therefore, the set $D=$ $\left\{e_{0}, e_{(n-1) / 2}, g_{(n+5) / 2}\right\}$ is the minimal doubly resolving set. Hence, Lemma 2 holds.

It is displayed from the whole technique that $\psi\left(R_{n}\right)=3$, for $n \geq 5$. The statement of the theorem by using Lemmas 1 and 2 is mentioned below.

Theorem 3. Let $R_{n}$ be the convex polytope; then, $\psi\left(R_{n}\right)=3$ for $n \geq 5$. 
TABLE 2: Vectors of metric coordinates for $R_{n}$ whenever $n$ is even, where $n \geq 5$.

\begin{tabular}{|c|c|c|}
\hline$j$ & $S_{j}\left(f_{0}\right)$ & $D=\left\{e_{0}, e_{(n-2) / 2}, g_{1}\right\}$ \\
\hline \multirow[t]{3}{*}{0} & $f_{0}$ & $(1,(n / 2), 2)$ \\
\hline & $e_{0}$ & $(0,((n-2) / 2), 2)$ \\
\hline & $e_{n-1}$ & $(1,(n / 2), 3)$ \\
\hline \multirow[t]{5}{*}{1} & $f_{1}$ & $(1,((n-2) / 2), 1)$ \\
\hline & $f_{n-1}$ & $(2,(n / 2), 3)$ \\
\hline & $g_{0}$ & $(2,((n+2) / 2), 1)$ \\
\hline & $e_{j-1}$ & $(j-1,((n-2 j) / 2), j)$ \\
\hline & $e_{n-j}$ & $(j,((n-2 j+2) / 2),((n+2 j-6) / 2))$ \\
\hline \multirow{6}{*}{$2 \leq j \leq((n-2) / 2)$} & $f_{j}$ & $(j,((n-2 j) / 2), j)$ \\
\hline & $f_{n-j}$ & $(j+1,((n-2 j+2) / 2),((n+2 j-6) / 2))$ \\
\hline & $g_{j-1}$ & $(j,((n-2 j+4) / 2), j-2)$ \\
\hline & $g_{n-j+1}$ & $(j+1,((n-2 j+6) / 2), j)$ \\
\hline & $e_{(n-2) / 2}$ & $(((n-2) / 2), 0,(n / 2))$ \\
\hline & $e_{n / 2}$ & $((n / 2), 1,((n+2) / 2))$ \\
\hline \multirow[t]{3}{*}{$n / 2$} & $f_{n / 2}$ & $((n / 2), 1,(n / 2))$ \\
\hline & $g_{(n-2) / 2}$ & $((n / 2), 2,((n-4) / 2))$ \\
\hline & $g_{(n+2) / 2}$ & $(((n+2) / 2), 3,(n / 2))$ \\
\hline
\end{tabular}

TABLE 3: Vectors of metric coordinates for $R_{n}$ whenever $n$ is odd, where $n \geq 9$.

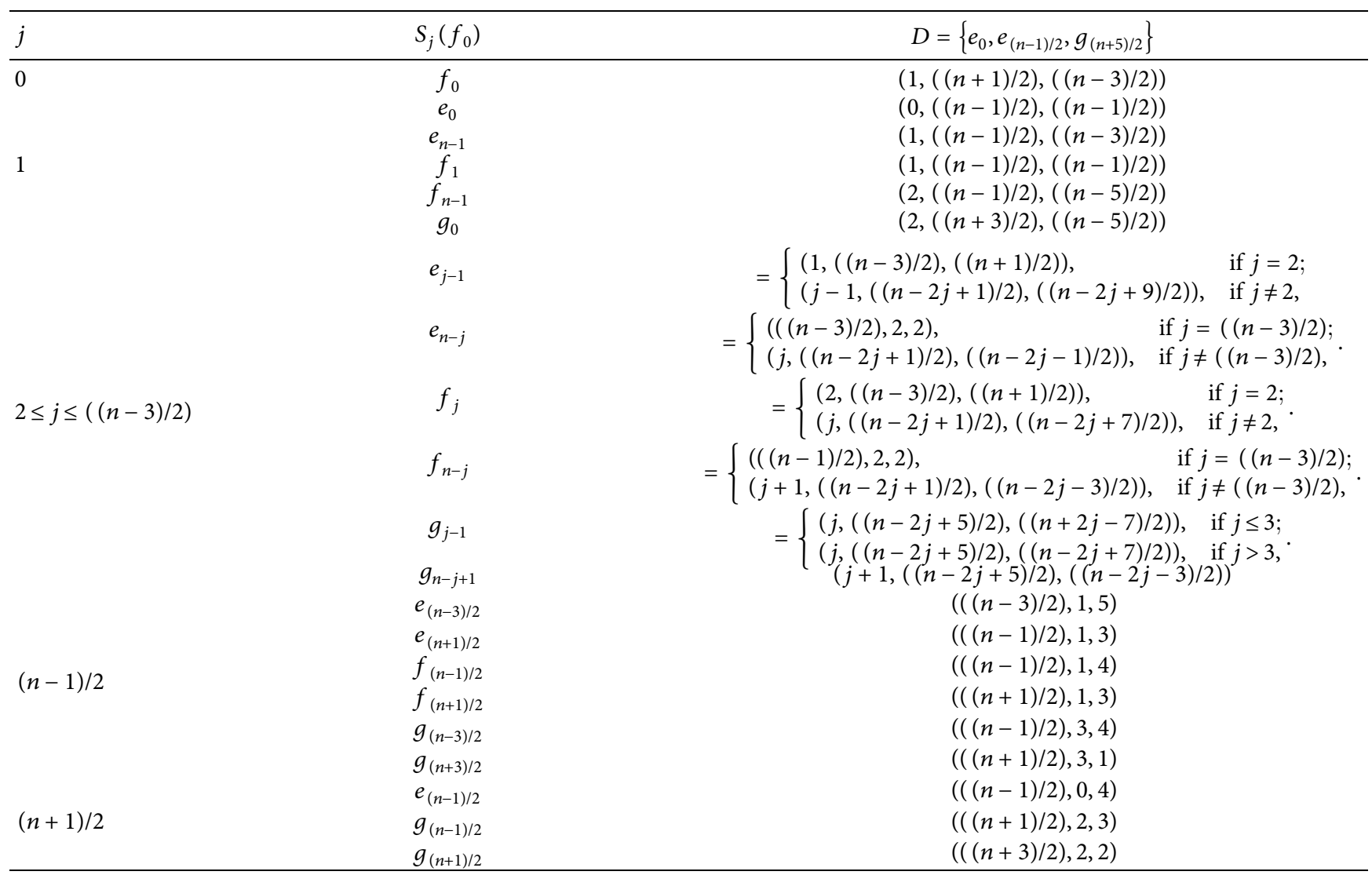

\section{The Double Metric Dimension for the Convex Polytope $Q_{n}$}

This section will particularly focus to find out the double metric dimension for convex polytope $Q_{n}$. The graph of convex polytope $Q_{n}$ consists of three-sided, four-sided, fivesided, and $n$-sided faces, as demonstrated in Figure 2.
We label the vertices of the inner cycle by $\left\{e_{j}: \forall 0 \leq\right.$ $j \leq n-1\}$, the vertices of the interior cycle by $\left\{f_{j}: \forall 0 \leq j \leq\right.$ $n-1\}$, the set of exterior vertices by $\left\{g_{j}: \forall 0 \leq j \leq n-1\right\}$, and the vertices of the outer cycle by $\left\{h_{j}: \forall 0 \leq j \leq n-1\right\}$, as demonstrated in Figure 2.

Theorem 2 implies that $\psi\left(Q_{n}\right) \geq 3$, for $n \geq 6$. Furthermore, we will show that $\psi\left(Q_{n}\right)=3$, for $n \geq 6$. 


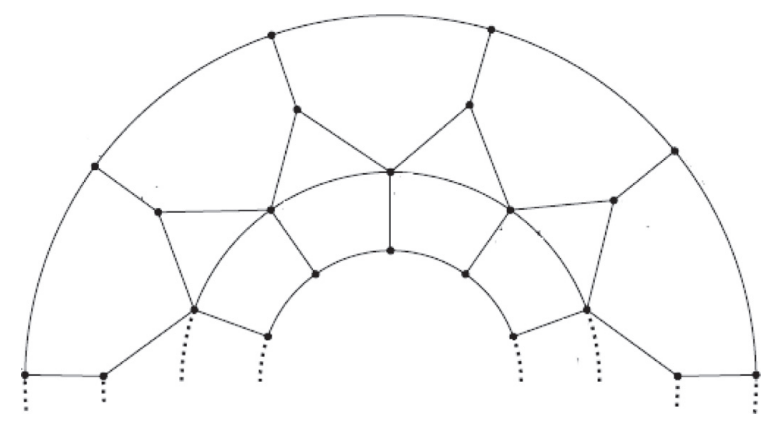

Figure 2: Convex polytope $Q_{n}$.

Now, to calculate distances for the convex polytope $Q_{n}$, define $S_{j}\left(f_{0}\right)=\left\{f \in V_{Q_{n}}: d\left(f_{0}, f\right)=j\right\}$ is the set of vertices in $V_{Q_{n}}$ at distance $j$ from $f_{0}$. For $\psi\left(Q_{n}\right)$ with $n \geq 6$, we can find the sets $S_{j}\left(f_{0}\right)$ that are elaborated in Table 4. Obviously, it can be seen from Figure 2 that $S_{j}\left(f_{0}\right)=\varnothing$, when $j>((n+2) / 2)$, for even $n$, and $S_{j}\left(f_{0}\right)=\varnothing$, when $j>((n+3) / 2)$, for odd $n$. Note that the sets $S_{j}\left(f_{0}\right)$ can clearly be used to find the distance between the two arbitrary vertices of $V_{Q_{n}}$ as follows.
The symmetry in Figure 2 shows that

$$
\begin{aligned}
d\left(f_{j}, f_{k}\right) & =d\left(e_{j}, e_{k}\right)=d\left(h_{j}, h_{k}\right) \\
& =d\left(f_{0}, f_{|j-k|}\right), \text { if } 0 \leq|j-k| \leq n-1, \\
d\left(f_{j}, e_{k}\right) & =d\left(f_{0}, e_{|j-k|}\right), \text { if } 0 \leq|j-k| \leq n-1 .
\end{aligned}
$$

For odd values of $n$, we have

$$
\begin{aligned}
& d\left(f_{j}, g_{k}\right)= \begin{cases}d\left(f_{0}, g_{|j-k|}\right)-1, & \text { if } 1 \leq|j-k| \leq \frac{n-1}{2} \text { for } j>k, \\
d\left(f_{0}, g_{|j-k|}\right)+1, & \text { if } \frac{n+1}{2} \leq|j-k| \leq n-1 \text { for } j>k, \\
d\left(f_{0}, g_{|j-k|}\right), & \text { if } 0 \leq|j-k| \leq n-1 \text { for } k \geq j,\end{cases} \\
& d\left(g_{j}, g_{k}\right)= \begin{cases}d\left(f_{0}, g_{|j-k|}\right)-1, & \text { if }|j-k|=0, \\
d\left(f_{0}, g_{|j-k|}\right), & \text { if } 1 \leq|j-k| \leq \frac{n-1}{2}, \\
d\left(f_{0}, g_{|j-k|}\right)+1, & \text { if } \frac{n+1}{2} \leq|j-k| \leq n-1, \\
d\left(f_{0}, e_{|j-k|}\right), & \text { if } 1 \leq|j-k| \leq \frac{n-1}{2} \text { for } j>k, \\
d\left(f_{0}, e_{|j-k|}\right)+1, & \text { if } \frac{n+1}{2} \leq|j-k| \leq n-1 \text { for } j>k, \\
d\left(f_{0}, e_{|j-k|}\right)+1, & \text { if } 0 \leq|j-k| \leq \frac{n-1}{2} \text { for } k \geq j, \\
d\left(f_{0}, e_{|j-k|}\right), & \text { if } \frac{n+1}{2} \leq|j-k| \leq n-1 \text { for } k>j,\end{cases}
\end{aligned}
$$


TABLe 4: $S_{j}\left(f_{0}\right)$ for $Q_{n}$.

\begin{tabular}{ccc}
\hline$n$ & $j$ & $S_{j}\left(f_{0}\right)$ \\
\hline & 1 & $\left\{e_{0}, f_{1}, f_{n-1}, g_{0}, g_{n-1}\right\}$ \\
& $2 \leq j \leq\lfloor n / 2\rfloor-1$ & $\left\{e_{j-1}, e_{n-j+1}, f_{j}, f_{n-j}, g_{j-1}, g_{n-j}, h_{j-2}, h_{n-j+1}\right\}$ \\
Even & $n / 2$ & $\left\{e_{(n-2) / 2}, e_{(n+2) / 2}, f_{n / 2}, g_{(n-2) / 2}, g_{n / 2}, h_{(n-4) / 2}, h_{(n+2) / 2}\right\}$ \\
& $(n+2) / 2$ & $\left\{e_{n / 2}, h_{(n-2) / 2}, h_{n / 2}\right\}$ \\
& $(n-1) / 2$ & \\
Odd & $(n+1) / 2$ & $\left\{e_{(n-3) / 2}, e_{(n+3) / 2}, f_{(n-1) / 2}, f_{(n+1) / 2}, g_{(n-3) / 2}, g_{(n+1) / 2}, h_{(n-5) / 2}, h_{(n+3) / 2}\right\}$ \\
& $(n+3) / 2$ & $\left\{e_{(n-1) / 2}, e_{(n+1) / 2}, g_{(n-1) / 2}, h_{(n-3) / 2}, h_{(n+1) / 2}\right\}$ \\
& & $\left\{h_{(n-1) / 2}\right\}$ \\
\hline
\end{tabular}

$$
d\left(g_{j}, h_{k}\right)= \begin{cases}d\left(f_{0}, g_{|j-k|}\right), & \text { if } 1 \leq|j-k| \leq \frac{n-1}{2} \text { for } j>k, \\ d\left(f_{0}, g_{|j-k|}\right)+1, & \text { if } \frac{n+1}{2} \leq|j-k| \leq n-1 \text { for } j>k, \\ d\left(f_{0}, g_{|j-k|}\right), & \text { if } 0 \leq|j-k| \leq \frac{n-1}{2} \text { for } k \geq j, \\ d\left(f_{0}, g_{|j-k|}\right)+1, & \text { if } \frac{n+1}{2} \leq|j-k| \leq n-1 \text { for } k>j, \\ d\left(f_{j}, h_{k}\right)= \begin{cases}d\left(f_{0}, h_{|j-k|}\right)-1, & \text { if } 1 \leq|j-k| \leq \frac{n-1}{2} \text { for } j>k, \\ d\left(f_{0}, h_{|j-k|}\right)+1, & \text { if } \frac{n+1}{2} \leq|j-k| \leq n-1 \text { for } j>k, \\ d\left(f_{0}, h_{|j-k|}\right), & \text { if } 0 \leq|j-k| \leq n-1 \text { for } k \geq j, \\ d\left(f_{0}, h_{|j-k|}\right), & \text { if } 1 \leq|j-k| \leq \frac{n-1}{2} \text { for } j>k, \\ d\left(f_{0}, h_{|j-k|}\right)+2, & \text { if } \frac{n+1}{2} \leq|j-k| \leq n-1 \text { for } j>k, \\ d\left(f_{0}, h_{|j-k|}\right)+1, & \text { if } 0 \leq|j-k| \leq n-1 \text { for } k \geq j .\end{cases} \end{cases}
$$

For even values of $n$, we have

$$
d\left(g_{j}, g_{k}\right)= \begin{cases}d\left(f_{0}, g_{|j-k|}\right)-1, & \text { if }|j-k|=0, \\ d\left(f_{0}, g_{|j-k|}\right), & \text { if } 1 \leq|j-k| \leq \frac{n-2}{2}, \\ d\left(f_{0}, g_{|j-k|}\right)+1, & \text { if } \frac{n}{2} \leq|j-k| \leq n-1,\end{cases}
$$


$d\left(f_{j}, g_{k}\right)= \begin{cases}d\left(f_{0}, g_{|j-k|}\right)-1, & \text { if } 1 \leq|j-k| \leq \frac{n-2}{2} \text { for } j>k, \\ d\left(f_{0}, g_{|j-k|}\right), & \text { if }|j-k|=\frac{n}{2} \text { for } j>k, \\ d\left(f_{0}, g_{|j-k|}\right)+1, & \text { if } \frac{n+2}{2} \leq|j-k| \leq n-1 \text { for } j>k, \\ d\left(f_{0}, g_{|j-k|}\right), & \text { if } 0 \leq|j-k| \leq n-1 \text { for } k \geq j,\end{cases}$

$d\left(e_{j}, g_{k}\right)= \begin{cases}d\left(f_{0}, e_{|j-k|}\right), & \text { if } 1 \leq|j-k| \leq \frac{n}{2} \text { for } j>k, \\ d\left(f_{0}, e_{|j-k|}\right)+1, & \text { if } \frac{n+2}{2} \leq|j-k| \leq n-1 \text { for } j>k, \\ d\left(f_{0}, e_{|j-k|}\right)+1, & \text { if } 0 \leq|j-k| \leq \frac{n-2}{2} \text { for } k \geq j, \\ d\left(f_{0}, e_{|j-k|}\right), & \text { if } \frac{n}{2} \leq|j-k| \leq n-1 \text { for } k>j,\end{cases}$

$d\left(f_{j}, h_{k}\right)= \begin{cases}d\left(f_{0}, h_{|j-k|}\right)-1, & \text { if } 1 \leq|j-k| \leq \frac{n-2}{2} \text { for } j>k, \\ d\left(f_{0}, h_{|j-k|}\right), & \text { if }|j-k|=\frac{n}{2} \text { for } j>k, \\ \left.d\left(f_{0}, h_{|j-k|}\right)\right)+1, & \text { if } \frac{n+2}{2} \leq|j-k| \leq n-1 \text { for } j>k, \\ d\left(f_{0}, h_{|j-k|}\right), & \text { if } 0 \leq|j-k| \leq n-1 \text { for } k \geq j,\end{cases}$

$d\left(g_{j}, h_{k}\right)= \begin{cases}d\left(f_{0}, g_{|j-k|}\right), & \text { if } 1 \leq|j-k| \leq \frac{n-2}{2} \text { for } j>k, \\ d\left(f_{0}, g_{|j-k|}\right)+1, & \text { if } \frac{n}{2} \leq|j-k| \leq n-1 \text { for } j>k, \\ d\left(f_{0}, g_{|j-k|}\right), & \text { if } 0 \leq|j-k| \leq \frac{n-2}{2} \text { for } k \geq j, \\ d\left(f_{0}, g_{|j-k|}\right)+1, & \text { if } \frac{n}{2} \leq|j-k| \leq n-1 \text { for } k>j,\end{cases}$

$d\left(e_{j}, h_{k}\right)= \begin{cases}d\left(f_{0}, h_{|j-k|}\right), & \text { if } 1 \leq|j-k| \leq \frac{n-2}{2} \text { for } j>k, \\ d\left(f_{0}, h_{|j-k|}\right)+1, & \text { if }|j-k|=\frac{n}{2} \text { for } j>k, \\ d\left(f_{0}, h_{|j-k|}\right)+2, & \text { if } \frac{n+2}{2} \leq|j-k| \leq n-1 \text { for } j>k, \\ d\left(f_{0}, h_{|j-k|}\right)+1, & \text { if } 0 \leq|j-k| \leq n-1 \text { for } k \geq j .\end{cases}$ 
TABLE 5: Vectors of metric coordinates for $Q_{n}$ whenever $n$ is even, where $n \geq 6$.

\begin{tabular}{|c|c|c|}
\hline$j$ & $S_{j}\left(f_{0}\right)$ & $D=\left\{e_{0}, e_{n / 2}, h_{0}\right\}$ \\
\hline \multirow[t]{3}{*}{0} & $f_{0}$ & $(1,((n+2) / 2), 2)$ \\
\hline & $e_{0}$ & $(0,(n / 2), 3)$ \\
\hline & $f_{1}$ & $(2,(n / 2), 3)$ \\
\hline \multirow[t]{6}{*}{1} & $f_{n-1}$ & $(2,((n-2) / 2), 1)$ \\
\hline & $g_{0}$ & $(2,((n+2) / 2), 1)$ \\
\hline & $g_{n-1}$ & $(2,((n+2) / 2), 2)$ \\
\hline & $e_{j-1}$ & $(j-1,((n-2 j+2) / 2), j+1)$ \\
\hline & $e_{n-j+1}$ & $(j-1,((n-2 j+2) / 2), j+2)$ \\
\hline & $f_{j}$ & $(j+1,((n-2 j+2) / 2), j+1)$ \\
\hline \multirow{8}{*}{$2 \leq j \leq((n-2) / 2)$} & $f_{n-j}$ & $(j+1,((n-2 j+2) / 2), j+2)$ \\
\hline & $g_{j-1}$ & $(j+1,((n-2 j+4) / 2), j)$ \\
\hline & $g_{n-j}$ & $(j+1,((n-2 j+4) / 2), j+1)$ \\
\hline & $h_{j-2}$ & $(j+1,((n-2 j+8) / 2), j-2)$ \\
\hline & $h_{n-j+1}$ & $(j+1,((n-2 j+8) / 2), j-1)$ \\
\hline & $e_{(n-2) / 2}$ & $(((n-2) / 2), 1,((n+2) / 2))$ \\
\hline & $e_{(n+2) / 2}$ & $(((n-2) / 2), 1,((n+4) / 2))$ \\
\hline & $f_{n / 2}$ & $(((n+2) / 2), 1,((n+2) / 2))$ \\
\hline \multirow[t]{5}{*}{$n / 2$} & $\mathcal{g}_{(n-2) / 2}$ & $(((n+2) / 2), 2,(n / 2))$ \\
\hline & $g_{n / 2}$ & $(((n+2) / 2), 2,((n+2) / 2))$ \\
\hline & $h_{(n-4) / 2}$ & $(((n+2) / 2), 4,((n-4) / 2))$ \\
\hline & $h_{(n+2) / 2}$ & $(((n+2) / 2), 4,((n-2) / 2))$ \\
\hline & $e_{n / 2}$ & $((n / 2), 0,((n+4) / 2))$ \\
\hline$(n+2) / 2$ & $h_{(n-2) / 2}$ & $(((n+4) / 2), 3,((n-2) / 2))$ \\
\hline & $h_{n / 2}$ & $(((n+4) / 2), 3,(n / 2))$ \\
\hline
\end{tabular}

TABLE 6: Vectors of metric coordinates for $Q_{n}$ whenever $n$ is odd, where $n \geq 13$.

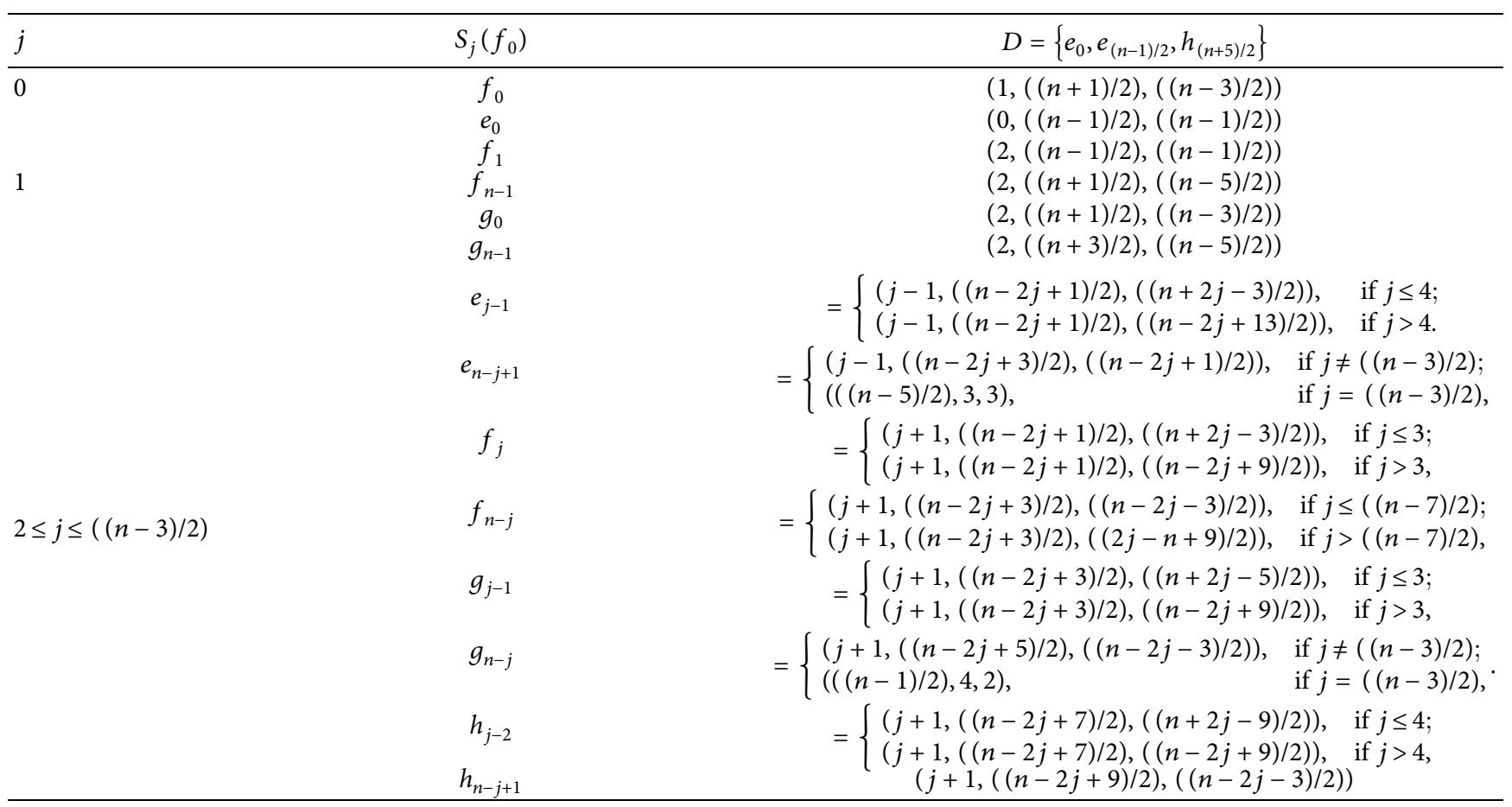


TABLE 6: Continued.

\begin{tabular}{lll}
\hline$j$ & $S_{j}\left(f_{0}\right)$ & $D=\left\{e_{0}, e_{(n-1) / 2}, h_{(n+5) / 2}\right\}$ \\
\hline & $e_{(n-3) / 2}$ & $(((n-3) / 2), 1,7)$ \\
& $e_{(n+3) / 2}$ & $(((n-3) / 2), 2,4)$ \\
& $f_{(n-1) / 2}$ & $(((n+1) / 2), 1,5)$ \\
& $f_{(n+1) / 2}$ & $(((n+1) / 2), 2,4)$ \\
& $g_{(n-3) / 2}$ & $(((n+1) / 2), 2,5)$ \\
& $g_{(n+1) / 2}$ & $(((n+1) / 2), 3,3)$ \\
& $h_{(n-5) / 2}$ & $(((n+1) / 2), 4,5)$ \\
& $h_{(n+3) / 2}$ & $(((n+1) / 2), 5,1)$ \\
& $e_{(n-1) / 2}$ & $(((n-1) / 2), 0,6)$ \\
& $e_{(n+1) / 2}$ & $(((n-1) / 2), 1,5)$ \\
& $g_{(n-1) / 2}$ & $(((n+3) / 2), 2,4)$ \\
& $h_{(n-3) / 2}$ & $(((n+3) / 2), 3,4)$ \\
& $h_{(n+1) / 2}$ & $(((n+3) / 2), 4,2)$ \\
& $h_{(n-1) / 2}$ & $(((n+5) / 2), 3,3)$ \\
\hline
\end{tabular}

As an outcome, if the distance $d\left(f_{0}, f\right)$ for every $f \in V_{Q_{n}}$ is known, then one can recreate the distances between any two vertices of $V_{\mathrm{Q}_{n}}$.

Lemma 3. $\psi\left(Q_{n}\right)=3$, for $n \geq 6$, whenever $n$ is even.

Proof. The representations of $Q_{n}$ according to the set $D=$ $\left\{e_{0}, e_{n / 2}, h_{0}\right\}$ are explained in Table 5 .

Now, from Table 5, the first metric coordinate of the vector of $f_{0} \in S_{j}\left(f_{0}\right)$ is equal to 1 . Similarly, it can be checked that there are no two vertices $p_{1}, p_{2} \in S_{j}\left(f_{0}\right)$, for every $j \in\{1,2, \ldots,((n+2) / 2)\}$ such as $r\left(p_{1}, D\right)-r\left(p_{2}, D\right)=0$. Thus, there are no two vertices $p_{1} \in S_{j}\left(f_{0}\right)$ and $p_{2} \in S_{k}\left(f_{0}\right)$, for any $j, k \in\{1,2, \ldots,((n+2) / 2)\}, j \neq k$, such as $r\left(p_{1}, D\right)-$ $r\left(p_{2}, D\right)=j-k$. Therefore, the set $D=\left\{e_{0}, e_{n / 2}, h_{0}\right\}$ is the minimal doubly resolving set. Thus, Lemma 3 holds.

Lemma 4. $\psi\left(Q_{n}\right)=3$, for $n \geq 6$, whenever $n$ is odd.

Proof. The minimal doubly resolving sets for $Q_{7}, Q_{9}$, and $Q_{11}$ are

$$
D= \begin{cases}\left\{e_{0}, e_{3}, h_{1}\right\}, & \text { if } n=7, \\ \left\{e_{0}, e_{4}, h_{7}\right\}, & \text { if } n=9, \\ \left\{e_{0}, e_{5}, h_{2}\right\}, & \text { if } n=11\end{cases}
$$

The representations of convex polytope $Q_{n}$ (where $n \geq 13)$ according to the set $D=\left\{e_{0}, e_{(n-1) / 2}, h_{(n+5) / 2}\right\}$ are explained in Table 6.

Now, from Table 6, the first metric coordinate of the vector of $f_{0} \in S_{j}\left(f_{0}\right)$ is equal to 1 . Similarly, it can be checked that there are no two vertices $p_{1}, p_{2} \in S_{j}\left(f_{0}\right)$, for every $j \in\{1,2, \ldots,((n+3) / 2)\}$ such as $r\left(p_{1}, D\right)-r\left(p_{2}\right.$, $D)=0$. Thus, there are no two vertices $p_{1} \in S_{j}\left(f_{0}\right)$ and $p_{2} \in S_{k}\left(f_{0}\right)$, for any $j, k \in\{1,2, \ldots,((n+3) / 2)\}, j \neq k$, such as $r\left(p_{1}, D\right)-r\left(p_{2}, D\right)=j-k$. Therefore, the set $D=\left\{e_{0}\right.$, $\left.e_{(n-1) / 2}, h_{(n+5) / 2}\right\}$ is the minimal doubly resolving set. Hence, Lemma 4 holds.
It is displayed from the whole technique that $\psi\left(Q_{n}\right)=3$, for $n \geq 6$. The statement of the theorem by using Lemmas 3 and 4 is mentioned below.

Theorem 4. Let $Q_{n}$ be the convex polytope, then $\psi\left(Q_{n}\right)=3$, for $n \geq 6$.

\section{Conclusion}

Despite of the fact that determining the minimal resolving sets of general graphs is computationally tough, the metric dimension has been gaining all the attention due to its applications in the different fields such as computer networking, navigation of robots, sonar technology, and optimization problems. The doubly resolving sets are a reasonable tool to successfully diagnose the source of infection within a network. The metric and double metric dimensions are NP-hard in general case.

The focus of article was the computation of the double metric dimension regarding convex polytopes $R_{n}$ and $Q_{n}$. We conclude that the double metric dimension of these classes of plane graphs is finite and independent of the number of vertices in these graphs. Just three vertices suitably picked up are sufficient to doubly resolve all the vertices of these classes of plane graphs.

\section{Data Availability}

All data used to support the findings of the study are included within the article.

\section{Conflicts of Interest}

The authors declare that they have no conflicts of interest.

\section{Authors' Contributions}

All authors contributed equally to this study. 


\section{Acknowledgments}

This research work was funded by Baoji Education Institute of Shaanxi, China, and University of Management and Technology, Pakistan.

\section{References}

[1] U. Ali, Y. Ahmad, and M. S. Sardar, "On 3-total edge product cordial labeling of tadpole, book and flower graphs," Open Journal of Mathematical Sciences, vol. 4, no. 1, pp. 48-55, 2020.

[2] F. Asif, Z. Zahid, and S. Zafar, "Leap zagreb and leap hyperzagreb indices of jahangir and jahangir derived graphs," Engineering and Applied Science Letter, vol. 3, no. 2, pp. 1-8, 2020.

[3] F. Harary and R. A. Melter, "On the metric dimension of a graph," Ars Combinatoria, vol. 2, pp. 191-195, 1976.

[4] P. J. Slater, "Leaves of trees," in Proceedings of the 6th Southeastern Conference on Combinatorics, Graph Theory and Computing, pp. 549-559, Boca Raton, FL, USA, February 1975.

[5] S. Khuller, B. Raghavachari, and A. Rosenfeld, "Landmarks in graphs," Discrete Applied Mathematics, vol. 70, no. 3, pp. 217-229, 1996.

[6] G. Chartrand, L. Eroh, M. A. Johnson, and O. R. Oellermann, "Resolvability in graphs and the metric dimension of a graph," Discrete Applied Mathematics, vol. 105, no. 1-3, pp. 99-113, 2000.

[7] P. Erdos and A. Renyi, "On two problems of information theory," Publications of the Mathematical Institute of the Hungarian Academy of Sciences, vol. 8, pp. 241-254, 1963.

[8] B. Lindstrom, "On a combinatory detection problem I," Publications of the Mathematical Institute of the Hungarian Academy of Sciences, vol. 9, pp. 195-207, 1964.

[9] V. Chvtal, "Mastermind," Combinatorica, vol. 3, pp. 325-329, 1983.

[10] K. Liu and N. Abu-Ghazaleh, "Virtual coordinates with backtracking for void traversal in geographic routing," in Lecture Notes on Computer Science, T. Kunz and S. S. Ravi, Eds., vol. 4104, pp. 46-59, Springer, Berlin, Germany, 2006.

[11] A. Sebö and E. Tannier, "On metric generators of graphs," Mathematics of Operations Research, vol. 29, no. 2, pp. 383393, 2004.

[12] Z. Beerliova, F. Eberhard, T. Erlebach et al., "Network discovery and verification," IEEE Journal on Selected Areas in Communications, vol. 24, no. 12, pp. 2168-2181, 2006.

[13] H. F. M. Salih, S. M. Mershkhan, and S. M. Mershkhan, "Generalized the liouville's and möbius functions of graph," Open Journal of Mathematical Sciences, vol. 4, no. 1, pp. 186-194, 2020.

[14] P. S. Buczkowski, G. Chartrand, C. Poisson, and P. Zhang, "On $k$-dimensional graphs and their bases," Periodica Mathematica Hungarica, vol. 46, no. 1, pp. 9-15, 2003.

[15] I. Tomescu and I. Javaid, "On the metric dimension of the Jahangir graph," Bulletin mathématique de la Société des Sciences Mathématiques de Roumanie, vol. 50, no. 98, pp. 371-376, 2007.

[16] M. Ali, G. Ali, A. Q. Baig, and M. K. Shafiq, "On the metric dimension of mobius ladders," Ars Combinatoria, vol. 105, pp. 403-410, 2012.

[17] M. Baca, E. T. Baskoro, A. N. M. Salman, S. W. Saputro, and D. Suprijanto, "The metric dimension of regular bipartite graphs," Bulletin mathématique de la Société des Sciences, vol. 54, pp. 15-28, 2011.
[18] M. Imran and A. Q. Baig, "A special class of convex polytopes with constant metric dimension," Journal of Combinatorial Mathematics and Combinatorial Computing, vol. 77, pp. 197-205, 2011.

[19] M. Imran, S. A. Ul Haq Bokhary, and A. Q. Baig, "On families of convex polytopes with constant metric dimension," Computers \& Mathematics with Applications, vol. 60, no. 9, pp. 2629-2638, 2010.

[20] I. Tomescu and M. Imran, " $R$-sets and metric dimension of necklace graphs," Applied Mathematics \& Information Sciences, vol. 9, no. 1, pp. 63-67, 2015.

[21] A. Ahmad, M. Bača, and S. Sultan, "Computing the metric dimension of kayak paddles graph and cycles with chord," Proyecciones (Antofagasta), vol. 39, no. 2, pp. 287-300, 2020.

[22] M. Numan, S. I. Butt, and A. Taimur, "Super cyclic antimagic covering for some families of graphs," Open Journal of Mathematical Sciences, vol. 5, no. 1, pp. 27-33, 2021.

[23] H. M. Nagesh and V. R. Girish, "On the entire zagreb indices of the line graph and line cut-vertex graph of the subdivision graph," Open Journal of Mathematical Sciences, vol. 4, no. 1, pp. 470-475, 2020.

[24] B. Spinelli, L. E. Celis, and P. Thiran, "How many sensors to localize the source?: the double metric dimension of random networks," in Proceedings of the 2018 56th Annual Allerton Conference on Communication, Control, and Computing (Allerton), pp. 1036-1043, Monticello, IL, USA, October 2018.

[25] J. Cáceres, C. Hernando, M. Mora et al., "On the metric dimension of cartesian products of graphs," SIAM Journal on Discrete Mathematics, vol. 21, no. 2, pp. 423-441, 2007.

[26] J. Kratica, M. Čangalović, and V. Kovačević-Vujčić, "Computing minimal doubly resolving sets of graphs," Computers \& Operations Research, vol. 36, no. 7, pp. 2149-2159, 2009.

[27] A. Ahmad and S. Sultan, "On minimal doubly resolving sets of circulant graphs," Acta Mechanica Slovaca, vol. 21, no. 1, pp. 6-11, 2017.

[28] M. Cangalovic, J. Kratica, V. Kovacevic-Vujcic, and M. Stojanovic, "Minimal doubly resolving sets of prism graphs," Optimization, vol. 62, pp. 1037-1043, 2013.

[29] J. Kratica, V. Kovacevic-Vujcic, M. Cangalovic, and M. Stojanovic, "Minimal doubly resolving sets and the strong metric dimension of hamming graphs," Applicable Analysis and Discrete Mathematics, vol. 6, no. 1, pp. 63-71, 2012.

[30] J. Kratica, V. Kovačević-Vujčić, M. Stojanović, and M. Stojanovic, "Minimal doubly resolving sets and the strong metric dimension of some convex polytopes," Applied Mathematics and Computation, vol. 218, no. 19, pp. 9790-9801, 2012.

[31] A. Ahmad, M. Baca, and S. Sultan, "On the minimal doubly resolving sets of harary graph," Acta Mathematica Universitatis Comenianae, vol. 89, no. 1, pp. 123-129, 2019.

[32] X. Chen, X. Hu, and C. Wang, "Approximation for the minimum cost doubly resolving set problem," Theoretical Computer Science, vol. 609, no. 3, pp. 526-543, 2016.

[33] M. Ahmad, Z. Zahid, and S. Zafar, "On minimal edge version of doubly resolving sets of a graph,” 2018, https://arxiv.org/ abs/1807.02365.

[34] M. Ahmad, N. Ameen, Z. Zahid, and S. Zafar, "Computing edge version of metric and double metric dimensions of kayak paddle graphs," Discrete Mathematics, Algorithms and Applications, vol. 12, no. 5, Article ID 2050070, 2020.

[35] M. Baca, "On magic labellings of convex polytopes," Annals Discrete Math, vol. 51, pp. 13-16, 1992.

[36] M. Imran, A. Q. Baig, and A. Ahmad, "Families of plane graphs with constant metric dimension," Utilitas Mathematica, vol. 88, pp. 43-57, 2012. 